\title{
Effects of Electron-Transfer Coupled with Collision-Induced Dissociation (ET/CID) on Doubly Charged Peptides and Phosphopeptides
}

\author{
Chih-Wei Liu, ${ }^{1}$ Chien-Chen Lai ${ }^{1,2}$ \\ ${ }^{1}$ Institute of Molecular Biology, National Chung Hsing University, No. 250, Kuo-Kuang Road, Taichung 402, Taiwan \\ ${ }^{2}$ Graduate Institute of Chinese Medical Science, China Medical University, Taichung, Taiwan
}

\begin{abstract}
Electron-transfer dissociation (ETD) is a useful peptide fragmentation technique that can be applied to investigate post-translational modifications (PTMs), the sequencing of highly hydrophilic peptides, and the identification of large peptides and even intact proteins. In contrast to traditional fragmentation methods, such as collision-induced dissociation (CID), ETD produces $\mathrm{c}$ - and $\mathrm{z}$-type product ions by randomly cleaving the $\mathrm{N}-\mathrm{C} \alpha$ bonds. The disappointing fragmentation efficiency of ETD for doubly charged peptides and phosphopeptide ions has been improved by ETcaD (supplemental activation). However, the ETD data derived from most database search algorithms yield low confidence scores due to the presence of unreacted precursors and charge-reduced ions within MS/MS spectra. In this work, we demonstrate that eight out of ten standard doubly charged peptides and phosphopeptides can be effortlessly identified by electron-transfer coupled with collision-induced dissociation (ET/CID) using the SEQUEST algorithm without further spectral processing. ET/CID was performed with the further dissociation of the charge-reduced ions isolated from ETD ion/ion reactions. ET/CID had high fragmentation efficiency, which elevated the confidence scores of doubly charged peptide and phosphospeptide sequencing. ET/CID was found to be an effective fragmentation strategy in "bottom-up" proteomic analysis.
\end{abstract}

Key words: Collision-induced dissociation, Electron-transfer dissociation, Doubly charged, Phosphopeptides, Peptides

\section{Introduction}

Tn the past decade, tandem mass spectrometry (MS/MS) 1 has played a major role in the bottom-up approach of peptide sequencing and protein identification [1-5]. The gas-

Electronic supplementary material The online version of this article (doi:10.1007/s13361-010-0020-9) contains supplementary material, which is available to authorized users.

Correspondence to: Chien-Chen Lai; e-mail: lailai@dragon.nchu.edu.tw phase ion dissociation or fragmentation of isolated precursor ions generates consecutive sequence-informative product ions and reveals the identities of the corresponding amino acid sequences. The unique primary sequence of the digested peptide reveals an exact protein ID through in silico database search algorithms. Collision-induced dissociation (CID) is the most widely used peptide fragmentation technique in tandem mass spectrometry [6-9]. The precursor ions are kinetically excited and activated by collision with the buffer gas. During the activation process, the internal energy of precursor ions is accumulated and converted into

Received: 26 July 2010

Revised: 13 October 2010

Accepted: 13 October 2010

Published online: 27 January 2011 
vibrational energy, which is distributed throughout all of the covalent bonds. The fragmentation occurs when the distributed vibrational energy exceeds the required activation energy for bond cleavage. After ion fragmentation via CID, a consecutive series of b- and y-type product ions are produced due to the relatively low activation energy of amide bond cleavage. Unfortunately, the presence of certain amino acids (e.g., Asp, Glu, and Pro residues) [10] and post-translational modifications (PTMs) (e.g., Ser/ Thr phosphorylations [11] and O-linked glycosylations) of the peptides can limit the sequence coverage provided by CID fragmentation.

Trypsin is the enzyme most commonly used in enzymatic protein digestion. Doubly charged protonated ions are dominantly produced following electrospray ionization (ESI) of tryptic peptides. Doubly charged protonated ions exhibit excellent fragmentation efficiency via CID [12]. However, the intense neutral-loss peaks and the relative absence of other product ions in CID spectra of phosphopeptides hamper the sequence identification of target phosphopeptides and the localization of phosphorylated sites (scheme 1, CID) [13, 14]. $\mathrm{MS}^{3}$ experiments are used to further activate the intense neutral-loss peaks, and to generate the sequence-informative fragment product ions. A multistage activation (MSA) strategy is also applied to reduce the duty cycle for phosphopeptide analysis [13].

Electron-based ion activation methods such as electroncapture dissociation (ECD) [15] and electron-transfer dissociation (ETD) [16] have been shown to be useful for investigating PTMs [16-20], sequencing highly hydrophilic peptides rich in Asp and Glu residues [20], and identifying large peptides and small proteins [20-22]. Electron-based ion activation methods are, therefore, complementary to CID in MS/MS sequencing analysis. When ECD is coupled with Fourier transform ion cyclotron resonance mass spectrometry (FT-ICR-MS), odd-electron protonated ion species are generated when multicharged peptide cations capture the thermal electrons. When ETD is coupled with ion trap-type mass spectrometry (IT-MS), the ion/ion reactions between protonated ions and radical anions (generally fluoranthene) produce unstable odd-electron protonated ion species. Both of those methods favor cleavage of the $\mathrm{N}-\mathrm{C} \alpha$ bonds, thus forming c- and z-type product ions.

In ETD spectra, the unreacted precursor and chargereduced ions produce major peaks in the product ion mass spectrum (Scheme 1, ETD). The charge-reduced ions are derived from precursor ions that receive at least one electron but do not dissociate further (ETnoD). This phenomenon is especially common in doubly charged precursor ions. Recently, Coon et al. demonstrated that supplemental activation (i.e., ETcaD) of the chargereduced ions provides a way to enhance the efficiency of ETD fragmentation for the sequencing of doubly charged precursor ions (Scheme 1, ETcaD) [23]. ETcaD can supply "low-energy" CID to disrupt the noncovalent interactions between the undissociated $\mathrm{c}$ and $\mathrm{z}$ ions $[23,24]$. This strategy generates ETD-specific product ions alone and improves sequence coverage. ETcaD has been employed to sequence doubly-charged peptide ions. However, in ETcaD, odd-electron $\mathrm{c}^{+\cdot}$ and even-electron $\mathrm{z}^{+}$ions are generated from the hydrogen transfer between the $c$ and $z$ ions [23]. The signals from the $\mathrm{c}^{+\cdot}$ and $\mathrm{z}^{+}$ions usually dominate the ETcaD spectra. In SEQUEST algorithms, only the c (even-electron) and $z$ (odd-electron) ions in ETD can be considered real product ions.

SEQUEST is one of the most important database search algorithms for tandem MS spectra [25-27]. SEQUEST was originally designed to query CID-generated MS/MS data. However, in response to the increasing number of researchers using electron-based activation techniques in large-scale proteomic analyses, SEQUEST was modified to allow searches for $\mathrm{c}$ (even-electron) and $\mathrm{z}$ (odd-electron) ions from ETD. The abundance of unreacted precursor and charge-reduced ions generated by ETD often suppress the peaks of product ions in ETD spectra. Even though ETcaD can eliminate the intense peaks from charge-reduced ions, this technique still results in a profusion of unreacted precursor ions. ETD data always yield low confidence scores, which results in the identification of fewer peptides using SEQUEST algorithms. Coon et al. showed that removing the unreacted precursor, the charge-reduced ions, and neutral losses from chargereduced ions from the ETD spectra ultimately led to an increase in the relative abundance of ETD product ions [28]. The number of unique identifications was increased using the open mass spectrometry search algorithm (OMSSA) [29]. Nevertheless, this modified data format is not compatible with the SEQUEST algorithm in routine analysis.

Wu and Karger et al. [30] demonstrated $\mathrm{MS}^{3}$ experiments where "low-energy" CID was used to further dissociate the charge-reduced ions generated from ETD. Such low-energy CID only disrupted the noncovalent interactions of the undissociated $\mathrm{c}$ and $\mathrm{z}$ ions and simplified the $\mathrm{MS}^{3}$ spectra, making it easy to locate PTM sites and determine the backbone. However, charge-reduced ions were still present in these $\mathrm{MS}^{3}$ spectra. Campbell et al. [31] performed ETD while simultaneously applying CID to doubly charged precursor ions in a single MS/MS experiment (ETD/CID). This ETD/CID approach increased sequence coverage, yielding more $\mathrm{b}$ and $\mathrm{y}$ ions through CID and $\mathrm{c}$ and $\mathrm{z}$ ions from ETD. However, the charge-reduced ions in the ETD/ CID spectra suppressed the relative abundances of the product ion peaks. Furthermore, Wu and Karger et al. [32] used "high-energy" CID for $\mathrm{MS}^{3}$ experiments to determine the disulfide linkages in recombinant therapeutic proteins through sequence-informative product ions $(b, y, c$, and $z$ ions, Scheme 2) from the highly charged precursor ions. The high-energy approach used in those $\mathrm{MS}^{3}$ experiments also played a key role in our study.

In this study, we first performed ETD reactions on doubly charged precursor ions (Scheme 1, ETD). The 
isolated charge-reduced ions resulting from ETD were subsequently dissociated with "high-energy" CID (Scheme 1, ET/CID). Those ET/CID MS ${ }^{3}$ spectra contained b, y, c and z product ions with high relative abundances. We then compared the Xcorr values of the CID, ETD, ETcaD, and ET/CID spectra using the SEQUEST algorithm. We found that ET/CID was an effective fragmentation strategy for identifying doubly charged peptides and phosphopeptides ions without the need for further spectral processing.

\section{Experimental}

\section{Chemicals and Reagents}

A standard mixture of phosphopeptides (cat. no. P33357) and MassPREP phosphopeptide standard enolase (cat. no. 186003285) were purchased from Invitrogen (USA) and Waters (USA), respectively. Acetonitrile (ACN) and formic acid (FA) were obtained from Merck (Germany) and Fluka (Switzerland), respectively. DI water was derived from a Barnstead Nanopure system (>18 M $\Omega$ ). The analytical standards were dissolved in $50 \%$ ACN with $0.1 \%$ formic acid to achieve final concentrations ranging from $0.5 \mu \mathrm{M}$ to $1 \mu \mathrm{M}$. The peptides were then directly infused into the linear ion trap mass spectrometer via an electrospray source (LTQ XL with ETD, Thermo Fisher Scientific, USA).

\section{Fragmentation}

The peptides were fragmented with CID, ETD, ETcaD, and ET/CID. MS/MS conditions for each of the fragmentation methods were as follows. For CID, we used the $\mathrm{MS}^{2}$ scan mode ( $\sim 330 \mathrm{MS} / \mathrm{scan})$, an activation time of $30 \mathrm{~ms}$, an activation $q_{\mathrm{u}}$ of 0.25 , and a normalized collision energy of $35 \%$. For ETD, we used the $\mathrm{MS}^{2}$ scan mode ( 400 MS/ scan). The reaction time with fluoranthene radical anions was $100 \mathrm{MS}$, and the anion automatic gain control (AGC) target value was $3 \times 10^{5}$. For $\mathrm{ETcaD}$, we used the $\mathrm{MS}^{2}$ scan mode ( $430 \mathrm{MS} / \mathrm{scan})$, the default $q_{\mathrm{u}}$ value of 0.15 , and a normalized collision energy of $12 \%$ for charge-reduced ions generated from precursor ions. For ET/CID MS ${ }^{3}$ experiments $[\sim 730 \mathrm{MS} / \mathrm{scan}(330 \mathrm{MS}+400 \mathrm{MS})]$, the isolated chargereduced ions from ETD were subsequently dissociated with "high-energy" CID (an activation $q_{\mathrm{u}}$ of 0.25 and a normalized collision energy of $35 \%$ were used). A different activation $q_{\mathrm{u}}$ value and normalized collision energy were used to further activate the charge-reduced ions generated from the ETD reaction. All of the MS/MS spectra were acquired in three microscans.

\section{SEQUEST Analysis}

The raw files (MS/MS spectra) were searched against a homemade database containing only the peptides employed in this study using the SEQUEST algorithm (BioworksBrowsor 3.3.1 SP1, Thermo Fisher Scientific).
Xcorr was used to compare the tandem mass spectra and amino acid sequence information in the database. The value of Xcorr was found to be independent of database size, but it was dependent on peptide size and charge state. The search parameters were as follows. Enzyme restriction was set to "no enzyme." The precursor mass tolerance was set at $\pm 2.0 \mathrm{Da}$, and the fragment ion mass tolerance was set at $\pm 0.5 \mathrm{Da}$. A low fragment ion mass tolerance was used to identify the unique c (even-electron) and $z^{\prime}$ (odd-electron) ions. The differential modification (phosphorylation) on STY residues was set at +97.96633 Da. In order to compare the Xcorr values (independent of database size) of the MS/MS spectra generated from the four MS/MS fragmentation methods, three groups of fragment ions $(\mathrm{B}+\mathrm{Y}+\mathrm{C}+\mathrm{Z}, \mathrm{B}+\mathrm{Y}$, and $\mathrm{C}+\mathrm{Z})$ were evaluated in each database search. $\mathrm{C}$ and $\mathrm{Z}$ corresponded to the $\mathrm{c}$ (even-electron) and $z^{\prime}$ (odd-electron) ions, respectively. To minimize the identification of false-positive product ions when evaluating product ion sequence coverage and peptide backbone cleavage efficiency, the product ions were counted and confirmed manually when the peak intensity was higher than $4 \%$ relative to that of the most intense matched product ion (excluding neutral-loss peaks). It has been reported that hydrogen atom transfer behavior between even-electron $\mathrm{c}$ ions and odd-electron $\mathrm{z}$. ions produces odd-electron $\mathrm{c}^{+\cdot}(1 \mathrm{Da}$ lighter than $\mathrm{c})$ and even-electron $\mathrm{z}^{+}$ions (1 Da heavier than $\mathrm{z}^{-}$) [23]. The SEQUEST algorithm interpreted even-electron $\mathrm{c}$ ions and odd-electron $z^{\cdot}$ ions as real product ions rather than $\mathrm{c}^{+}$. ions and $\mathrm{z}^{+}$ions. In this study, we focused on evenelectron $\mathrm{c}$ ions and odd-electron $\mathrm{z}^{\cdot}$ ions.

\section{Results and Discussion}

In this study, three unmodified peptides and seven phosphopeptides were examined. The sequences of these peptides are listed in Table 1. In order to simplify peptide annotations within this study, we gave a name to each peptide; for example, T18 indicates NVPL[pY]K, where $[\mathrm{pY}]$ indicates a phophorylated residue. The MS/MS data were generated from peptides activated by CID, ETD, and ETcaD. The charge-reduced ions generated from the ETD were subsequently dissociated with CID (ET/CID) to produce the $\mathrm{MS}^{3}$ data. All spectra were searched using SEQUEST algorithm, and Xcorr values were acquired for the corresponding spectra. There were two major reasons for choosing the SEQUEST algorithm to evaluate the quality of the spectra: (1) SEQUEST is often used for ITMS coupled with ETD; (2) the Xcorr value for a specific spectrum was independent of the size of the database.

\section{CID, ETD, and ETcaD of Doubly Charged Precursor Ions}

In the CID analysis of doubly charged peptides, 6 of the 10 peptides had Xcorr values smaller than $2.2(\mathrm{~B}+\mathrm{Y}$ ion 
Table 1. Information on the ten candidate peptides analyzed and the Xcorr values of the four fragmentation types obtained using the SEQUEST algorithm

\begin{tabular}{|c|c|c|c|c|c|c|c|c|}
\hline \multirow{2}{*}{$\begin{array}{l}\text { Peptide } \\
\text { name }\end{array}$} & \multirow{2}{*}{$\begin{array}{c}{[\mathrm{M}+2 \mathrm{H}]^{2+}} \\
\quad(\mathrm{m} / \mathrm{z})\end{array}$} & \multirow{2}{*}{$\begin{array}{c}{[\mathrm{M}+2 \mathrm{H}]^{+\bullet}} \\
(\mathrm{m} / \mathrm{z})\end{array}$} & \multirow{2}{*}{$\begin{array}{c}\text { Fragmentation } \\
\text { type }\end{array}$} & \multirow{2}{*}{$\begin{array}{l}\text { Precursor } \\
\text { ion }(m / z)\end{array}$} & \multicolumn{3}{|c|}{ Xcorr value $^{\mathrm{b}}$} & \multirow[t]{2}{*}{ Peptide sequences ${ }^{\mathrm{d}}$} \\
\hline & & & & & $\mathrm{B}+\mathrm{Y}+\mathrm{C}+\mathrm{Z}$ ions & $\mathrm{B}+\mathrm{Y}$ ions & $\mathrm{C}+\mathrm{Z}$ ions & \\
\hline \multirow[t]{4}{*}{$\mathrm{T} 18$} & \multirow[t]{4}{*}{407.20} & \multirow[t]{4}{*}{814.40} & CID & 407.20 & 1.150 & 1.160 & $\mathrm{NM}^{\mathrm{c}}$ & \multirow[t]{4}{*}{ NVPL $[p Y] K$} \\
\hline & & & ETD & 407.20 & 1.387 & 0.647 & 0.735 & \\
\hline & & & ETcaD & 407.20 & 1.271 & NM & 0.839 & \\
\hline & & & $\mathrm{ET} / \mathrm{CID}^{\mathrm{a}}$ & 814.40 & 1.771 & 1.002 & 0.790 & \\
\hline \multirow[t]{4}{*}{ T19 } & \multirow[t]{4}{*}{432.21} & \multirow[t]{4}{*}{864.42} & CID & 432.21 & 0.753 & 0.723 & NM & \multirow[t]{4}{*}{ HLADL[pS]K } \\
\hline & & & ETD & 432.21 & 1.855 & NM & 1.690 & \\
\hline & & & ETcaD & 432.21 & 2.044 & NM & 1.959 & \\
\hline & & & ET/CID & 864.42 & 2.173 & 1.157 & 1.007 & \\
\hline \multirow[t]{4}{*}{ A2 } & \multirow[t]{4}{*}{523.77} & \multirow{4}{*}{1047.54} & CID & 523.77 & 1.195 & 1.215 & NM & \multirow[t]{4}{*}{ DRVYIHPF } \\
\hline & & & ETD & 523.77 & 0.947 & NM & 0.937 & \\
\hline & & & ETcaD & 523.77 & 1.843 & NM & 1.783 & \\
\hline & & & $\mathrm{ET} / \mathrm{CID}$ & 1047.54 & 2.288 & 0.921 & 1.373 & \\
\hline \multirow[t]{4}{*}{ A1 } & \multirow[t]{4}{*}{648.85} & \multirow[t]{4}{*}{1297.70} & CID & 648.85 & 2.198 & 2.039 & 0.178 & \multirow[t]{4}{*}{ DRVYIHPFHL } \\
\hline & & & ETD & 648.85 & 1.284 & 0.413 & 1.202 & \\
\hline & & & ETcaD & 648.85 & 1.963 & 0.288 & 2.020 & \\
\hline & & & ET/CID & 1297.70 & 3.399 & 1.366 & 2.240 & \\
\hline \multirow{4}{*}{ T431P } & 684.84 & 1369.68 & CID & 684.84 & 4.969 & 4.856 & 0.216 & VNQIG[pT]LSESIK \\
\hline & & & ETD & 684.84 & 0.666 & 0.027 & 0.637 & \\
\hline & & & ETcaD & 684.84 & 1.932 & 0.151 & 1.943 & \\
\hline & & & ET/CID & 1369.68 & 3.766 & 1.140 & 2.816 & \\
\hline T432P & 724.82 & 1449.64 & CID & 724.82 & 4.331 & 4.019 & 0.482 & VNQIGTL[pS]E[pS]IK \\
\hline & & & ETD & 724.82 & 0.267 & 0.141 & 0.128 & \\
\hline & & & ETcaD & 724.82 & 2.245 & 0.738 & 1.905 & \\
\hline & & & ET/CID & 1449.64 & 3.500 & 1.553 & 2.384 & \\
\hline MBP104 & 789.93 & 1579.86 & CID & 789.93 & 2.006 & 1.740 & NM & GKGRGLSLSRFSWGA \\
\hline & & & ETD & 789.93 & 0.703 & 0.015 & 0.685 & \\
\hline & & & ETcaD & 789.93 & 1.349 & 0.209 & 1.179 & \\
\hline & & & $\mathrm{ET} / \mathrm{CID}$ & 1579.86 & 2.747 & 0.729 & 2.083 & \\
\hline MAPK177 & 835.34 & 1670.68 & CID & 835.34 & 5.230 & 5.283 & NM & DHTGFL[pT]E[pY]VATR \\
\hline & & & ETD & 835.34 & 0.040 & 0.047 & NM & \\
\hline & & & ETcaD & 835.34 & 2.018 & NM & 1.992 & \\
\hline & & & $\mathrm{ET} / \mathrm{CID}$ & 1670.68 & 5.728 & 3.125 & 2.994 & \\
\hline IR1142 & 851.88 & 1703.76 & CID & 851.88 & 2.864 & 2.829 & 0.210 & TRDI[pY]ETDYYRK \\
\hline & & & ETD & 851.88 & 0.122 & NM & 0.146 & \\
\hline & & & ETcaD & 851.88 & 0.968 & 0.180 & 0.784 & \\
\hline & & & ET/CID & 1703.76 & 4.154 & 2.067 & 2.571 & \\
\hline pTp & 860.95 & 1721.90 & CID & 860.95 & 0.270 & 0.289 & NM & VPIPGRFDRRV[pT]VE \\
\hline & & & ETD & 860.95 & 0.820 & 0.356 & 0.462 & \\
\hline & & & ETcaD & 860.95 & 1.884 & 0.287 & 1.593 & \\
\hline & & & ET/CID & 1721.90 & 3.315 & 0.981 & 2.385 & \\
\hline
\end{tabular}

${ }^{\mathrm{a}} \mathrm{ET} / \mathrm{CID}$ was $\mathrm{MS}^{3}$ fragmentation, in other words the charge-reduced ions generated from ETD were dissociated with CID (activation $q_{\mathrm{u}}$ of 0.25 , normalized collision energy of $35 \%$ ). The ion isolation width was $m / z 50$ for the $\mathrm{MS}^{3}$ step

${ }^{b}$ Xcorr value was calculated with three groups of product ions

${ }^{\mathrm{c}} N M$ no match using this product ion group

${ }^{\mathrm{d}}$ Phosphoserine, phosphothreonine, and phosphotyrosine are abbreviated to $p S, p T$, and $p Y$, respectively

types, Table 1). In general, 2.2 was a relatively low criterion for identifying doubly charged peptides [33]. There were proline residues within the peptide sequences for peptides $\mathrm{A} 2$ and $\mathrm{A} 1$, and the peptide bonds at the $\mathrm{N}$ termini of the proline residues were easy to fragment via CID. The other peptide bonds were not fragmented (T18 and A2). Boersema et al. reported a similar dissociation pattern for phosphoserine and phosphothreonine peptides [13]. Neutral losses of phosphate groups from phosphopeptides, $[\mathrm{M}+2 \mathrm{H}-98]^{2+}$, were significantly stronger than $\mathrm{b}$ and $\mathrm{y}$ ions (T19 and $\mathrm{pTp}$ ). The Xcorr values of those spectra did not always satisfy the criteria due to the relatively low abundance of product ions in the CID spectra. Using $\mathrm{B}+\mathrm{Y}+\mathrm{C}+\mathrm{Z}$ ions to evaluate Xcorr values, we found that the values were similar to the $\mathrm{B}+\mathrm{Y}$ ion types due to fewer product ions of $\mathrm{c}$ and $\mathrm{z}^{\prime}$ ions. Therefore, six of the ten peptides were not identified by CID fragmentation. In the ETD analysis of doubly charged peptides, no peptides or phosphopeptides could be identified because the Xcorr values were much smaller than $2.2(\mathrm{C}+\mathrm{Z}$ ion types). ETD exhibited low efficiency at dissociating doubly charged peptides; the relative intensities of the $\mathrm{c}$ and $\mathrm{z}^{\circ}$ ions were less than $5 \%$. Unreacted precursor and charge-reduced ions were the dominant peaks in the ETD spectra of doubly charged peptides; therefore, ETD fragmentation does not produce adequate MS/MS spectra and sufficiently high Xcorr values to pass the criteria.

To improve the power of ETD to dissociate doubly charged ions, supplemental activation was used to disrupt 
noncovalent binding between $\mathrm{c}$ and $\mathrm{z}^{+\cdot}$ ions. Xcorr values of all ETcaD spectra were higher than ETD; however, the values did not satisfy the criteria. ETcaD gave lower charge-reduced ion intensities and more product ions than ETD. Although there was less interference with chargereduced ions based on the results of the database search, there was still an overabundance of unreacted precursor ions. Xcorr values of ETcaD spectra were not sufficient to identify all 10 doubly charged peptides and phosphopeptides $(\mathrm{C}+\mathrm{Z}$ ion types, Table 1$)$. Based on our results, doubly charged peptides and phosphopeptides could not be identified directly in MS/MS spectra generated from ETD and ETcaD.

\section{ET/CID of Doubly Charged Precursor Ions}

$\mathrm{Wu}$ and Karger et al. [30] demonstrated charge-reduced-CID $\left(\mathrm{MS}^{3}\right)$ fragmentation for highly charged precursor ions. They applied "low-energy" CID (normalized collision energy of $10 \%$ and an activation $q_{\mathrm{u}}$ of 0.15 ) to further dissociate charge-reduced ions from ETD. In their report, highly charged precursor ions from Lys-C digestion were generated
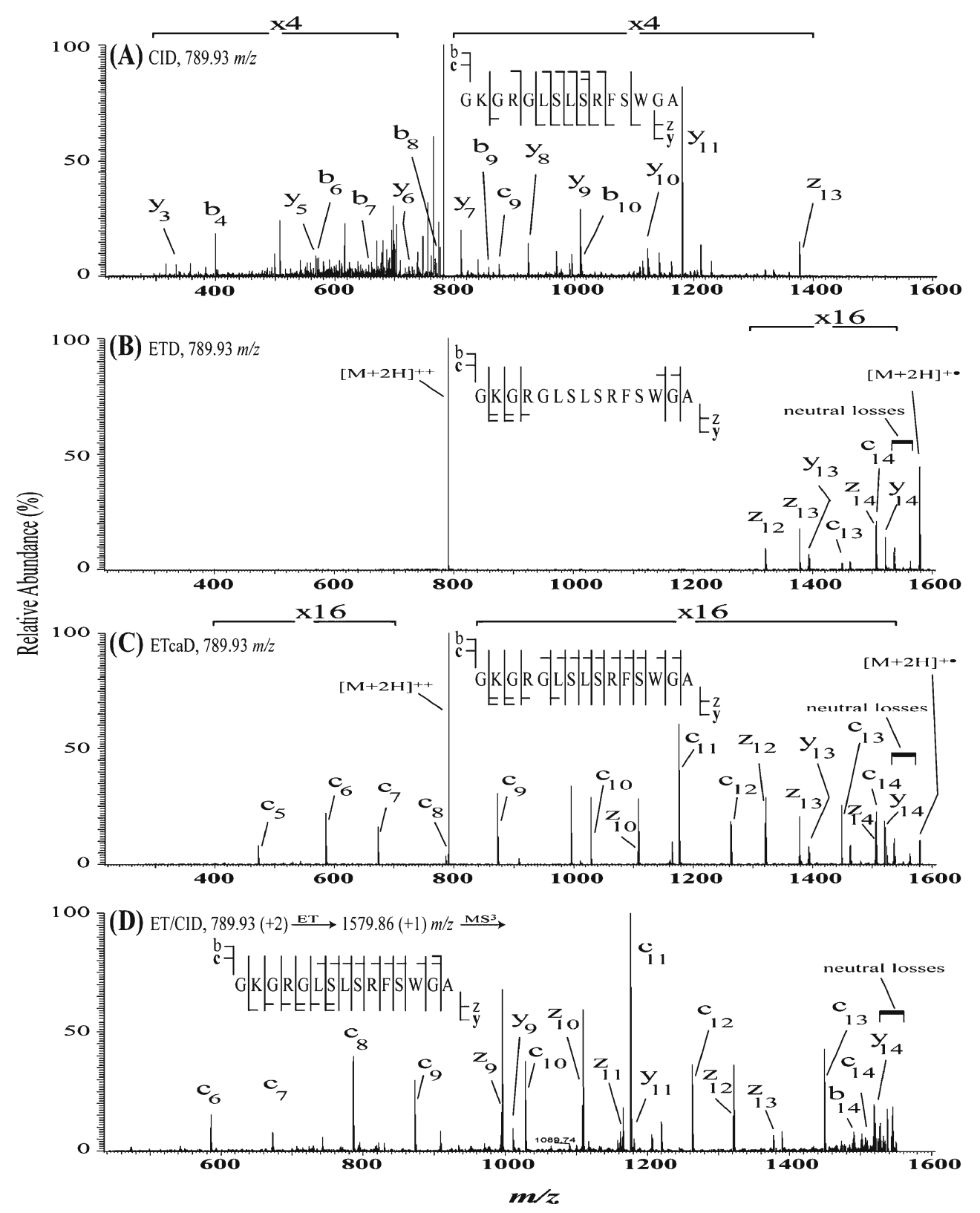

Figure 1. CID (A), ETD (B), ETcaD (C), and ET/CID (D) spectra of product ions from the doubly charged peptide MBP104 (GKGRGLSLSRFSWGA). Note that the $c$ and $z$ labels shown in the spectra indicate the even-electron $c$ ions and odd-electron $z^{\prime}$ ions, respectively 
in electrospray ionization. Due to the "low-energy" CID, the charge-reduced ions in the $\mathrm{MS}^{3}$ spectra still produced interference with product ions. Coon et al. [23] utilized ETcaD $\mathrm{MS}^{2}$ fragmentation, applying a normalized collision energy of $20 \%$ and an activation $q_{\mathrm{u}}$ value of 0.18 to disrupt charge-reduced ions and near-exclusively generate $\mathrm{c}$ and $\mathrm{z}$ ions (including $\mathrm{c}^{+\cdot}$ and $\mathrm{z}^{+}$ions). The unreacted precursor ions in the ETcaD spectra strongly suppressed the peaks of product ions. Campbell et al. [31] performed ETD while simultaneously applying CID to doubly charged precursor ions in a single MS/MS experiment (ETD/CID). The ETD/ $\mathrm{CID}$ approach increased the sequence coverage of $\mathrm{b}$ and $\mathrm{y}$ ions derived from precursor ions without affecting the number of $\mathrm{c}$ and $\mathrm{z}$ product ions. ETD/CID improved the sequence coverage of doubly charged ions and eliminated the abundance of unreacted precursor ions that are normally generated by ETD. ETD/CID, however, could not remove the charge-reduced ions that inhibit the relative intensities of the product ions. Moreover, the number of ETD-derived product ions (c and $z$ ions) was not increased through the CID of even-electron precursor ions.

ET/CID experiments were conducted in the $\mathrm{MS}^{3}$ scan mode to increase the relative abundances of product ions in the MS spectra by eliminating the influence of unreacted



Figure 2. CID (A), ETD (B), ETcaD (C), and ET/CID (D) spectra of product ions from the doubly charged phosphopeptide pTp (VPIPGRFDRRV $V_{P}$ TVE). Note that the $c$ and $z$ labels shown in the spectra indicate the even-electron $c$ ions and odd-electron $z^{*}$ ions, respectively. The neutral loss of phosphate is also shown 
precursor and charge-reduced ions. These ET/CID spectra were used to directly identify doubly charged peptides and phosphopeptides without further spectral processing. We, therefore, applied a normalized collision energy of $35 \%$ and a $q_{\mathrm{u}}$ value of 0.25 to activate the isolated charge-reduced ions generated from ETD reactions, and found that b, y, c, and $\mathrm{z}$ product ions were displayed in a single ET/CID spectrum. Using the SEQUEST search algorithm (Xcorr values $>2.2, \mathrm{C}+\mathrm{Z}$ ion types), we found that six of the ten standard peptides could be identified by ET/CID. By combining the $\mathrm{b}$ and $\mathrm{y}$ product ion results, eight peptides were recognized ( $\mathrm{B}+\mathrm{Y}+\mathrm{C}+\mathrm{Z}$ ion types). A larger number of peptides were identified via ET/CID than via CID, ETD and ETcaD (Table 1). A total of four peptides (A1, A2, MBP104 and pTp) were identified via ET/CID. The CID, ETD, ETcaD, and ET/CID spectra of MBP104 are shown in Figure 1. The enhanced relative intensities of product ions in the ET/CID spectra of MBP104 resulted in an Xcorr value of 2.747 (Figure 1d and Table 1). We could not directly identify MBP104 using CID, ETD, and ETcaD because of their low Xcorr values. CID, ETD, ETcaD, and ET/CID spectra of pTp are shown in Figure 2. pTp produced intense peaks from neutral losses of phosphate groups in the CID spectra, resulting in an Xcorr value of 0.270 (Figure 2a and Table 1 ). However, $\mathrm{pTp}$ was easily identified (Xcorr value $=$ $3.315)$ by ET/CID without any spectral processing (Figure 2d and Table 1). Neutral losses of phosphate groups were indeed observed in the ET/CID spectra due to the fragmentation of the charge-reduced even-electron ions $\left([\mathrm{M}+\mathrm{H}]^{+}\right)$derived from ETD side reactions (proton transfer without dissociation) [11]. However, a number of product ions with high relative intensities were also generated, which provided a wealth of sequence information. It was proposed that both $\mathrm{b}$ and $\mathrm{y}$ ions were generated from the charge-reduced even-electron ions ([M $+\mathrm{H}]^{+}$, Scheme 1 and 2) [32]. We were able to directly identify eight out of ten standard peptides and phosphopeptides due to the clarity of the ET/CID spectra without spectral processing $(\mathrm{B}+\mathrm{Y}+\mathrm{C}+\mathrm{Z}$ ion types).

Moreover, the effects of normalized collision energy (C) and activation $q_{\mathrm{u}}(\mathrm{Q})$ on the Xcorr values of the ET/CID spectra were examined (Table S1 of the Electronic Supplementary Material, ESM). The Xcorr values obtained using high-energy ET/CID (C35Q0.25) were significantly higher than those obtained using $\mathrm{C} 12 \mathrm{Q} 0.15$ and $\mathrm{C} 15 \mathrm{Q} 0.15$, due to the charge-reduced ions that remain when using low-energy ET/CID. In high-energy ET/CID spectra (C35Q0.25), the new $\mathrm{b}$ and $\mathrm{y}$ ions produced through the activation of the charge-reduced ions (even electron) enhanced the Xcorr values. Therefore, these data clearly show that ET/CID (C35Q0.25) is a powerful strategy that could be used in "bottom-up" proteomics to identify doubly charged peptides and phosphopeptides without further spectral processing. It had been reported that hydrogen atom transfer between even-electron $\mathrm{c}$ ions and odd-electron $\mathrm{z}$ ions produced oddelectron $\mathrm{c}^{+\cdot}(1 \mathrm{Da}$ lighter than $\mathrm{c})$ and even-electron $\mathrm{z}^{+}$ions (1 Da heavier than $\mathrm{z}$ ) [23]. The effect of $\mathrm{c}^{+\cdot}$ and $\mathrm{z}^{+}$ions upon the Xcorr values was also evaluated (Table S2 of the ESM). A higher mass tolerance of product ions $( \pm 1.0 \mathrm{Da})$ was used to include the contribution from the $\mathrm{H}$-transfer product ions $\left(\mathrm{c}^{+\cdot}\right.$ and $\mathrm{z}^{+}$ions). However, this did not lead to any obvious

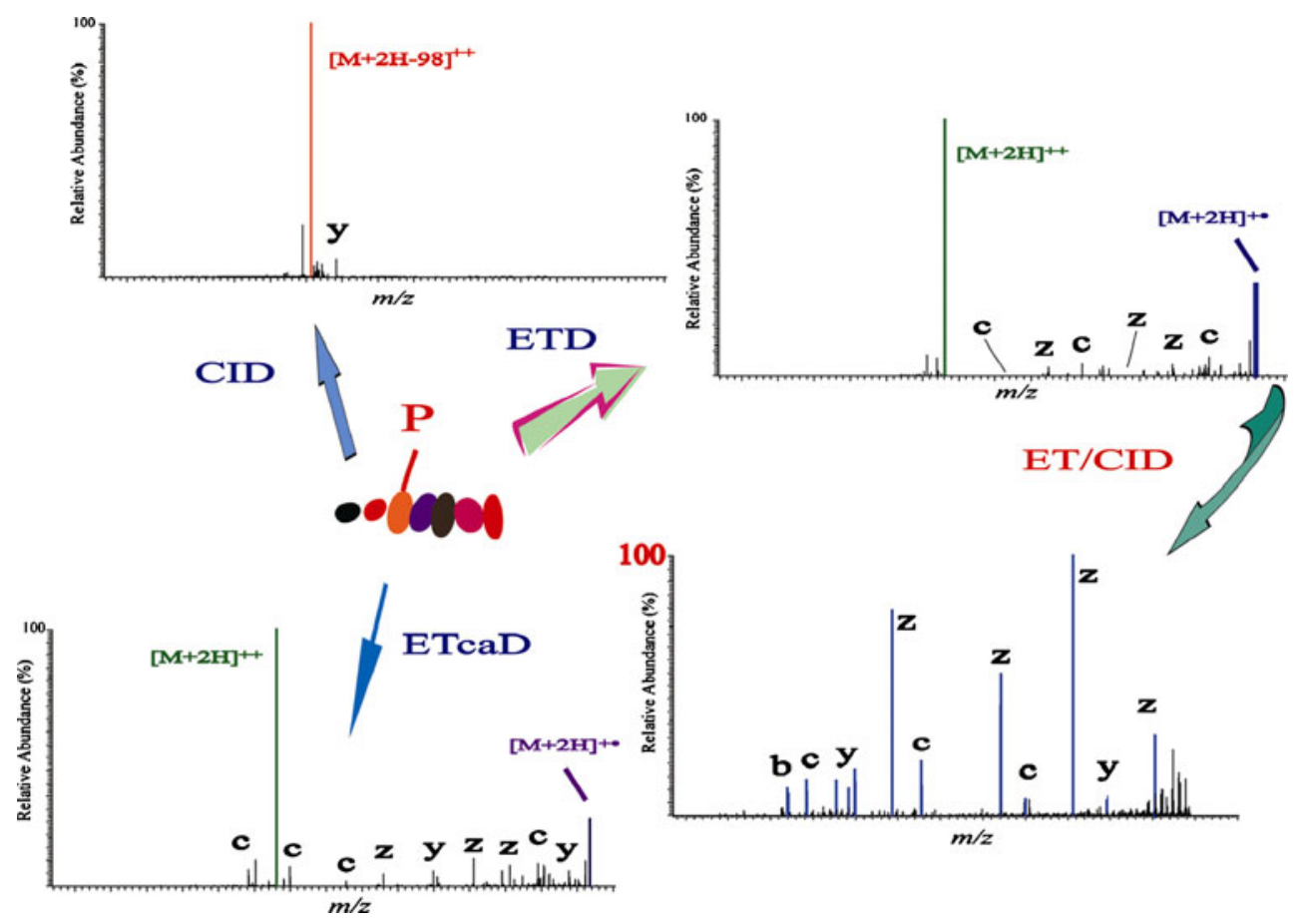

Scheme 1. Doubly charged phosphopeptides were activated by CID, ETD, ETcaD, and ET/CID. $P$ indicates a phosphate group 


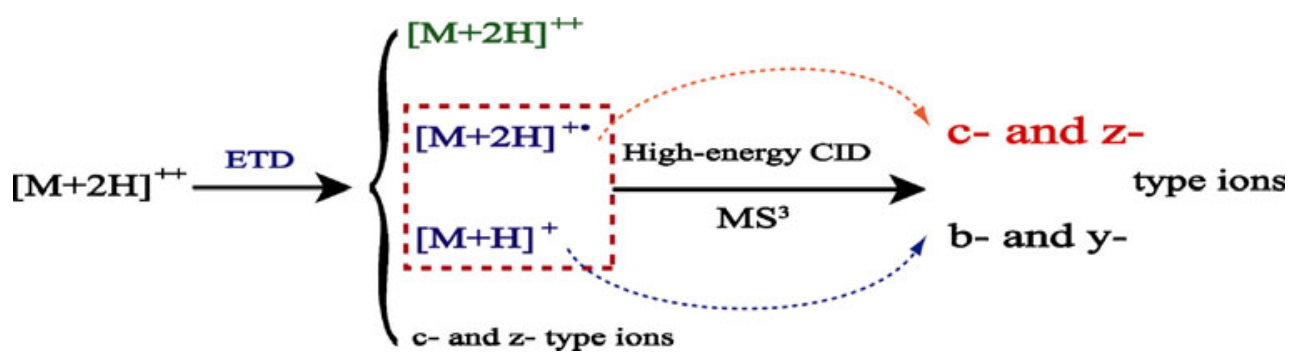

Scheme 2. The procedure used in our ET/CID MS ${ }^{3}$ experiments. First, ETD was applied to the doubly charged precursor ions, and then the isolated charge-reduced ions were dissociated with "high-energy" CID. High relative intensities of product ions were observed in the resulting ET/CID spectra

enhancement of the Xcorr values. The use of a higher mass tolerance of product ions caused an increased risk of random matches during database searching.

\section{Fragmentation Efficiency Calculations}

Fragmentation efficiency was calculated in order to evaluate the confidence scores of matched peptide sequences. The fragmentation efficiency was derived from the product ion sequence coverage and the peptide backbone cleavage efficiency. Proline linkages were also calculated, although the $\mathrm{N}$-terminus of proline was not cleaved by the ETD reaction.

Product ion sequence coverage (Table 2) was defined as

$$
\frac{\text { no. of observed product ion }}{\text { no. of theoretical product ion }} \times 100
$$

The peptide backbone cleavage efficiency (Table 3) was defined as

$$
\frac{\text { no. of observed backbone cleavages }}{\text { no. of peptide backbone bonds }} \times 100
$$

In ET/CID, the low-mass cutoff (LMCO) was increased because this isolates the larger $\mathrm{m} / \mathrm{z}$ values of the chargereduced ions for $\mathrm{MS}^{3}$ activation. The higher $\mathrm{LMCO}$ resulted in the loss of low $\mathrm{m} / \mathrm{z}$ product ions. Even though ET/CID utilized a higher LMCO for product ion detection in ion-trap mass spectrometry, ET/CID still led to high product ion sequence coverage (47.8\%), while ETD and ETcaD yielded $25.4 \%$ and $44.5 \%$, respectively ( $\mathrm{C}+\mathrm{Z}$ ion types, Table 2$)$. ET/CID also led to higher product ion sequence coverage of $\mathrm{B}+\mathrm{Y}+\mathrm{C}+\mathrm{Z}$ ions $(41.2 \%)$ than observed for the other three fragmentation methods in a single spectrum $(22.8 \%, 19.4 \%$, and $29.0 \%$, respectively). The LMCO problem could be solved by using other types of mass spectrometry equipped with ETD source (for example triple quadrupole ETD). Interestingly, for B $+\mathrm{Y}$ ions, we observed that $\mathrm{b}$ and $\mathrm{y}$ ions were also produced by ETD and ETcaD (13.3\% and 13.4\%, respectively). In particular, y ions were constantly generated in electron-based activation techniques due to the C-terminal residues of Arg and Lys derived from trypsin digestion. These data supported our reasoning behind increasing rather than decreasing the number of $\mathrm{b}$ and $\mathrm{y}$ product ions. The efficiency of ET/CID at cleaving $\mathrm{B}+\mathrm{Y}+\mathrm{C}+\mathrm{Z}$ ions in the peptide backbone was $91.4 \%$ (Table 3). In addition, the efficiency of ET/CID at cleaving $\mathrm{C}+$ $\mathrm{Z}$ ions in backbone bonds was $84.4 \%$. Therefore, ET/CID exhibited a high fragmentation efficiency that led to enhanced product ion sequence coverage for doubly charged peptides and phosphopeptides with the SEQUEST algorithm.

\section{Evaluation by a Realistic Database}

$\mathrm{dCn}(\mathrm{deltaCn})$ values - normalized scores obtained by calculating the difference between the first- and the second-ranked

\begin{tabular}{|c|c|c|c|c|c|c|c|c|c|c|c|c|}
\hline \multirow{2}{*}{$\begin{array}{c}\text { Peptide } \\
\text { name }\end{array}$} & \multicolumn{4}{|c|}{$\mathrm{B}+\mathrm{Y}+\mathrm{C}+\mathrm{Z}$ ions } & \multicolumn{4}{|c|}{$\mathrm{B}+\mathrm{Y}$ ions } & \multicolumn{4}{|c|}{$\mathrm{C}+\mathrm{Z}$ ions } \\
\hline & CID & ETD & ETcaD & ET/CID & CID & ETD & ETcaD & ET/CID & CID & ETD & ETcaD & $\mathrm{ET} / \mathrm{CID}$ \\
\hline $\mathrm{T} 18$ & 10.0 & 45.0 & 35.0 & 35.0 & 20.0 & 40.0 & 30.0 & 40.0 & 0.0 & 50.0 & 40.0 & 30.0 \\
\hline T19 & 45.8 & 33.3 & 33.3 & 50.0 & 66.7 & 8.3 & 8.3 & 50.0 & 25.0 & 58.3 & 58.3 & 50.0 \\
\hline A2 & 10.7 & 14.3 & 28.6 & 64.3 & 21.4 & 0.0 & 7.1 & 64.3 & 0.0 & 28.6 & 50.0 & 64.3 \\
\hline A1 & 16.7 & 13.9 & 22.2 & 44.4 & 33.3 & 5.6 & 0.0 & 38.9 & 0.0 & 22.2 & 44.4 & 50.0 \\
\hline T431P & 31.8 & 11.4 & 22.7 & 34.1 & 59.1 & 9.1 & 4.6 & 13.6 & 4.6 & 13.6 & 40.9 & 54.6 \\
\hline $\mathrm{T} 432 \mathrm{P}$ & 22.7 & 15.9 & 31.8 & 36.4 & 40.9 & 13.6 & 27.3 & 27.3 & 4.6 & 18.2 & 36.4 & 45.5 \\
\hline MBP104 & 28.6 & 12.5 & 28.6 & 32.1 & 50.0 & 7.1 & 7.1 & 14.3 & 7.1 & 17.9 & 50.0 & 50.0 \\
\hline MAPK177 & 39.6 & 25.0 & 35.4 & 56.3 & 75.0 & 33.3 & 16.7 & 54.2 & 4.2 & 16.7 & 54.2 & 58.3 \\
\hline IR1142 & 18.2 & 6.8 & 25.0 & 38.6 & 36.4 & 4.6 & 13.6 & 36.4 & 0.0 & 9.1 & 36.4 & 40.9 \\
\hline $\mathrm{pTp}$ & 3.9 & 15.4 & 26.9 & 21.2 & 7.7 & 11.5 & 19.2 & 7.7 & 0.0 & 19.2 & 34.6 & 34.6 \\
\hline Average & 22.8 & 19.4 & 29.0 & 41.2 & 41.1 & 13.3 & 13.4 & 34.7 & 4.5 & 25.4 & 44.5 & 47.8 \\
\hline
\end{tabular}

Table 2. Product ion sequence coverages (\%) of the four fragmentation types for the ten candidate peptides 
Table 3. Peptide backbone cleavage efficiencies $(\%)$ of the four fragmentation types for the ten candidate peptides

\begin{tabular}{|c|c|c|c|c|c|c|c|c|c|c|c|c|}
\hline \multirow{2}{*}{$\begin{array}{l}\text { Peptide } \\
\text { name }\end{array}$} & \multicolumn{4}{|c|}{$\mathrm{B}+\mathrm{Y}+\mathrm{C}+\mathrm{Z}$ ions } & \multicolumn{4}{|c|}{$\mathrm{B}+\mathrm{Y}$ ions } & \multicolumn{4}{|c|}{$\mathrm{C}+\mathrm{Z}$ ions } \\
\hline & CID & ETD & ETcaD & $\mathrm{ET} / \mathrm{CID}$ & CID & ETD & ETcaD & $\mathrm{ET} / \mathrm{CID}$ & CID & ETD & ETcaD & ET/CID \\
\hline $\mathrm{T} 18$ & 20.0 & 100.0 & 100.0 & 80.0 & 20.0 & 80.0 & 60.0 & 80.0 & 0.0 & 80.0 & 80.0 & 60.0 \\
\hline $\mathrm{T} 19$ & 100.0 & 100.0 & 100.0 & 83.3 & 100.0 & 16.6 & 16.7 & 83.3 & 33.3 & 100.0 & 100.0 & 83.3 \\
\hline $\mathrm{A} 2$ & 28.6 & 57.1 & 85.7 & 100.0 & 28.6 & 0.0 & 14.3 & 100.0 & 0.0 & 57.1 & 85.7 & 85.7 \\
\hline A1 & 44.4 & 44.4 & 77.8 & 100.0 & 44.4 & 11.1 & 0.0 & 66.7 & 0.0 & 44.4 & 77.8 & 88.9 \\
\hline $\mathrm{T} 431 \mathrm{P}$ & 72.7 & 36.4 & 81.8 & 100.0 & 72.7 & 18.2 & 9.1 & 27.3 & 9.1 & 27.3 & 81.8 & 100.0 \\
\hline $\mathrm{T} 432 \mathrm{P}$ & 54.6 & 45.5 & 72.7 & 90.9 & 45.5 & 27.3 & 45.5 & 45.5 & 9.1 & 36.4 & 72.7 & 81.8 \\
\hline MBP104 & 64.3 & 35.7 & 92.9 & 100.0 & 57.1 & 14.3 & 14.3 & 28.6 & 14.3 & 35.7 & 92.9 & 92.9 \\
\hline MAPK 177 & 83.3 & 75.0 & 91.7 & 100.0 & 83.3 & 66.7 & 33.3 & 91.7 & 8.3 & 33.3 & 91.7 & 100.0 \\
\hline IR1142 & 54.6 & 27.3 & 72.7 & 90.9 & 54.6 & 9.1 & 27.3 & 63.6 & 0.0 & 18.2 & 72.7 & 81.8 \\
\hline pTp & 7.7 & 53.9 & 76.9 & 69.2 & 7.7 & 15.4 & 30.8 & 15.4 & 0.0 & 38.5 & 69.2 & 69.2 \\
\hline Average & 53.0 & 57.5 & 85.2 & 91.4 & 51.4 & 25.9 & 25.1 & 60.2 & 7.4 & 47.1 & 82.5 & 84.4 \\
\hline
\end{tabular}

sequences - were derived. The $\mathrm{dCn}$ values were dependent on database size, sequence homology, and search parameters (mass tolerance, enzyme restrictions). To search against a realistic protein database, an enzyme restriction (e.g., to trypsin) must be employed to avoid random matches in "bottom-up" proteomics. In this study, four out of six tryptic peptides (T431p, T432p, MAPK177, and IR1142) were successfully identified using ET/ CID. The $\mathrm{dCn}$ values of those spectra were obtained by searching against a realistic human protein database $(39,423$ entries, Table 4), and the search parameters mimicked a general "bottom-up" proteomics strategy. The mass tolerances of the precursor and fragment ions were set to $\pm 2.0 \mathrm{Da}$ and $\pm 0.5 \mathrm{Da}$, respectively. The enzyme restriction was set to trypsin, and two miscleavages were available. Carbamidomethyl cysteine was set as a fixed modification, while other modifications were set as variable modifications. More search result data are shown in Table S3 of the ESM. We observed that a higher Xcorr value did not always translate into a better match based on $\mathrm{dCn}$ values.
Based on the Xcorr and $\mathrm{dCn}$ values, all of the peptides were easy to identify in ET/CID spectra (Table 4). Moreover, the phosphorylated site was clearly apparent in the ET/CID spectra of IR1142 (Table S3 of the ESM). Those data strongly indicate that ET/CID spectra yield specific results for trypsin-based "bottom-up" proteomics.

\section{Comparison of ETcaD (With Spectral Processing) and ET/CID (Without Spectral Processing)}

ETcaD data were processed manually using spectral processing methods [28] in order to remove the peaks from unreacted precursor ions, charge-reduced ions, and neutral losses from charge-reduced ions. The resulting dta files were then searched with the SEQUEST algorithm, and higher Xcorr values were obtained for these new ETcaD spectra (see Table S4 of the ESM). The Xcorr values of all ion types $(\mathrm{B}+\mathrm{Y}+\mathrm{C}+\mathrm{Z}, \mathrm{B}+\mathrm{Y}$, and $\mathrm{C}+\mathrm{Z}$ ions $)$ were

Table 4. Trypsin-dependent dCn evaluations of four tryptic peptides using a realistic human protein database (with 39423 entries) and the SEQUEST algorithm

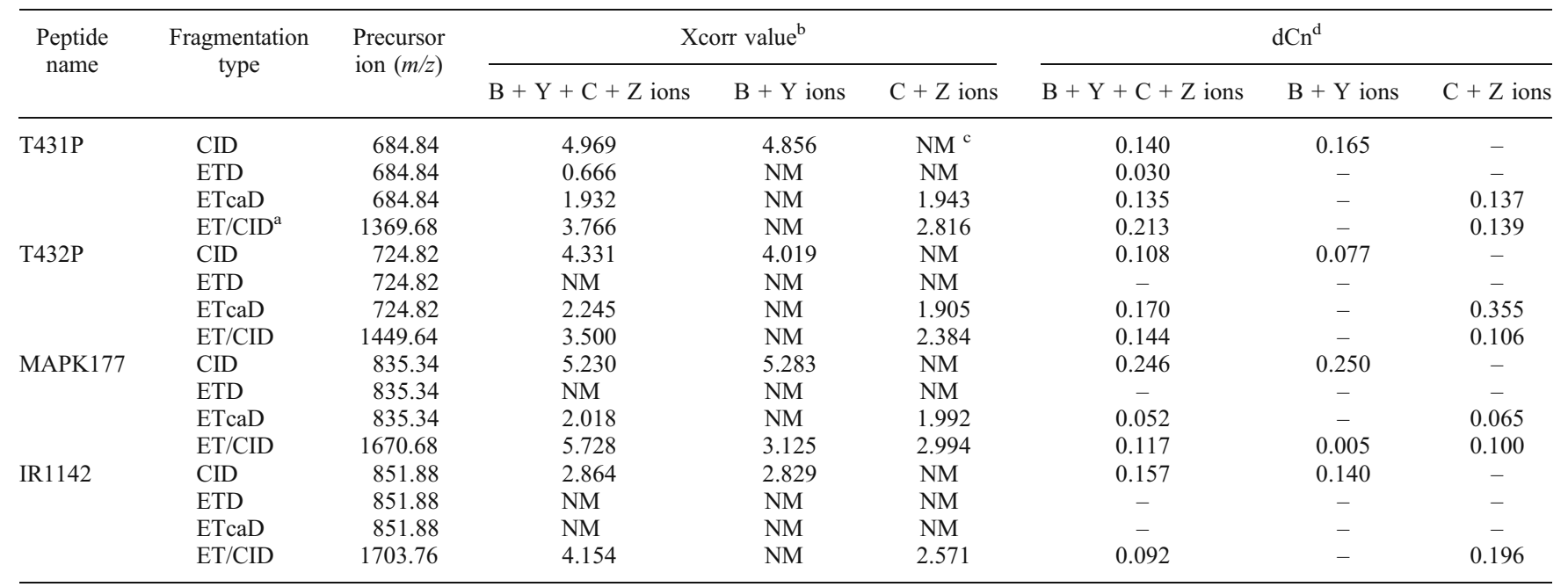

${ }^{\mathrm{a}} \mathrm{ET} / \mathrm{CID}$ was $\mathrm{MS}^{3}$ fragmentation, in other words, the charge-reduced ions generated from ETD were dissociated with CID (activation $q_{\mathrm{u}}$ of 0.25 , normalized collision energy of $35 \%$ ). The ion isolation width was $\mathrm{m} / z 50$ for the $\mathrm{MS}^{3}$ step

${ }^{\mathrm{b}}$ Xcorr value was calculated with three groups of product ions

${ }^{\mathrm{c}} N M$ no match using this product ion group

$\mathrm{d}_{\mathrm{dCn}}(\mathrm{deltaCn})$ was calculated with three groups of product ions 
enhanced due to the elevated relative abundances of the product ions. However, the Xcorr values obtained using the ET/CID data were still higher than those obtained with the processed ETcaD data, due to the abundance of $\mathrm{B}+\mathrm{Y}$ ions.

\section{Conclusions}

ETcaD is widely used to sequence doubly charged peptide ions, and it improves the utility of ETD in "bottom-up" proteomics. Although ETD complements CID in MS/MS peptide sequencing analysis, the abundance of unreacted precursor and charge-reduced ions generated by ETD often suppress the peaks of product ions from doubly charged peptides in ETD spectra. Therefore, without further spectral processing, it is difficult to identify doubly charged peptide sequences by ETD and ETcaD. Most of the peptides chosen for this study were doubly charged phosphopeptides, which are notorious for producing poor ETD spectra. Eight out of the ten standard doubly charged peptides and phosphopeptides were identified by ET/CID directly using the SEQUEST algorithm without spectral processing. ET/CID conducted in the $\mathrm{MS}^{3}$ scan mode increased the relative abundances of product ions by minimizing the influence of unreacted precursor and charge-reduced ions. The ET/CID data were conveniently analyzed using the SEQUEST algorithm. ET/CID exhibited a high fragmentation efficiency that assisted in peptide sequencing and PTM site location for doubly charged peptides and phosphopeptides.

\section{Acknowledgment}

The study was funded by a grant from the National Research Council of the Republic of China.

\section{References}

1. Bogdanov, B., Smith, R.D.: Proteomics by FTICR mass spectrometry: top down and bottom up. Mass Spectrom. Rev. 24, 168-200 (2005)

2. Wysocki, V.H., Resing, K.A., Zhang, Q., Cheng, G.: Mass spectrometry of peptides and proteins. Methods 35, 211-222 (2005)

3. Domon, B., Aebersold, R.: Mass spectrometry and protein analysis. Science 312, 212-217 (2006)

4. Mann, M., Kelleher, N.L.: Precision proteomics: the case for high resolution and high mass accuracy. Proc. Natl. Acad. Sci. U. S. A. 105, 18132-18138 (2008)

5. Griffin, T.J., Aebersold, R.: Advances in proteome analysis by mass spectrometry. J. Biol. Chem. 276, 45497-45500 (2001)

6. Griffin, T.J., Xie, H., Bandhakavi, S., Popko, J., Mohan, A., Carlis, J.V., Higgins, L.: iTRAQ reagent-based quantitative proteomic analysis on a linear ion trap mass spectrometer. J. Proteome Res. 6, 4200-4209 (2007)

7. Molina, H., Matthiesen, R., Kandasamy, K., Pandey, A.: Comprehensive comparison of collision induced dissociation and electron transfer dissociation. Anal. Chem. 80, 4825-4835 (2008)

8. Wells, J.M., McLuckey, S.A.: Collision-induced dissociation (CID) of peptides and proteins. Meth. Enzymol. 402, 148-185 (2005)

9. Aebersold, R., Mann, M.: Mass spectrometry-based proteomics. Nature 422, 198-207 (2003)

10. Qin, J., Chait, B.T.: Preferential fragmentation of protonated gas phase peptide ions adjacent to acidic amino acid residues. J. Am. Chem. Soc. 117, 5411-5412 (1995)

11. Schroeder, M.J., Shabanowitz, J., Schwartz, J.C., Hunt, D.F., Coon, J.J.: A neutral loss activation method for improved phosphopeptide sequence analysis by quadrupole ion trap mass spectrometry. Anal. Chem. 76, 3590-3598 (2004)

12. Good, D.M., Wirtala, M., McAlister, G.C., Coon, J.J.: Performance characteristics of electron transfer dissociation mass spectrometry. Mol. Cell. Proteomics 6, 1942-1951 (2007)

13. Boersema, P.J., Mohammed, S., Heck, A.J.: Phosphopeptide fragmentation and analysis by mass spectrometry. J. Mass Spectrom. 44, 861-878 (2009)

14. DeGnore, J.P., Qin, J.: Fragmentation of phosphopeptides in an ion trap mass spectrometer. J. Am. Soc. Mass Spectrom. 9, 1175-1188 (1998)

15. Zubarev, R.A., Kelleher, N.L., McLafferty, F.W.: Electron capture dissociation of multiply charged protein cations - a nonergodic process. J. Am. Chem. Soc. 120, 3265-3266 (1998)

16. Syka, J.E., Coon, J.J., Schroeder, M.J., Shabanowitz, J., Hunt, D.F.: Peptide and protein sequence analysis by electron transfer dissociation mass spectrometry. Proc. Natl. Acad. Sci. U. S. A 101, 9528-9533 (2004)

17. Molina, H., Horn, D.M., Tang, N., Mathivanan, S., Pandey, A.: Global proteomic profiling of phosphopeptides using electron transfer dissociation tandem mass spectrometry. Proc. Natl. Acad. Sci. U. S. A 104, 2199-2204 (2007)

18. Hogan, J.M., Pitteri, S.J., Chrisman, P.A., McLuckey, S.A.: Complementary structural information from a tryptic N-linked glycopeptide via electron transfer ion/ion reactions and collision-induced dissociation. $J$. Proteome Res. 4, 628-632 (2005)

19. Chi, A., Huttenhower, C., Geer, L.Y., Coon, J.J., Syka, J.E.P., Bai, D. L., Shabanowitz, J., Burke, D.J., Troyanskaya, O.G., Hunt, D.F.: Analysis of phosphorylation sites on proteins from Saccharomyces cerevisiae by electron transfer dissociation (ETD) mass spectrometry. Proc. Natl. Acad. Sci. U. S. A. 104, 2193-2198 (2007)

20. Domon, B., Bodenmiller, B., Carapito, C., Hao, Z., Huehmer, A., Aebersold, R.: Electron transfer dissociation in conjunction with collision activation to investigate the Drosophila melanogaster phosphoproteome. J. Proteome Res. 8, 2633-2639 (2009)

21. Bunger, M.K., Cargile, B.J., Ngunjiri, A., Bundy, J.L., Stephenson Jr., J.L.: Automated proteomics of E. coli via top-down electron-transfer dissociation mass spectrometry. Anal. Chem. 80, 1459-1467 (2008)

22. Kelleher, N.L.: Top-down proteomics. Anal. Chem. 76, 197A-203A (2004)

23. Swaney, D.L., McAlister, G.C., Wirtala, M., Schwartz, J.C., Coon, J.J.: Supplemental activation method for high-efficiency electrontransfer dissociation of doubly protonated peptide precursors. Anal. Chem. 79, 477-485 (2007)

24. Xia, Y., Han, H., McLuckey, S.A.: Activation of intact electron-transfer products of polypeptides and proteins in cation transmission mode ion/ ion reactions. Anal. Chem. 80, 1111-1117 (2008)

25. Eng, J.K., McCormack, A.L., Yates, J.R.I.: An approach to correlate tandem mass spectral data of peptides with amino acid sequences in a protein database. J. Am. Soc. Mass Spectrom. 5, 976-989 (1994)

26. Nesvizhskii, A.I., Vitek, O., Aebersold, R.: Analysis and validation of proteomic data generated by tandem mass spectrometry. Nat. Meth. 4, 787-797 (2007)

27. Kandasamy, K., Pandey, A., Molina, H.: Evaluation of several MS/MS search algorithms for analysis of spectra derived from electron transfer dissociation experiments. Anal. Chem. 81, 7170-7180 (2009)

28. Good, D.M., Wenger, C.D., McAlister, G.C., Bai, D.L., Hunt, D.F., Coon, J.J.: Post-acquisition ETD spectral processing for increased peptide identifications. J. Am. Soc. Mass Spectrom. 20, 1435-1440 (2009)

29. Geer, L.Y., Markey, S.P., Kowalak, J.A., Wagner, L., Xu, M., Maynard, D.M., Yang, X., Shi, W., Bryant, S.H.: Open mass spectrometry search algorithm. J. Proteome Res.3, 958-964 (2004)

30. Wu, S.L., Huhmer, A.F., Hao, Z., Karger, B.L.: On-Line LC-MS approach combining collision-induced dissociation (CID), electrontransfer dissociation (ETD), and CID of an isolated charge-reduced species for the trace-level characterization of proteins with posttranslational modifications. J. Proteome Res. 6, 4230-4244 (2007)

31. Campbell, J.L., Hager, J.W., Le Blanc, J.C.: On performing simultaneous electron transfer dissociation and collision-induced dissociation on multiply protonated peptides in a linear ion trap. J. Am. Soc. Mass Spectrom. 20, 1672-1683 (2009)

32. Wu, S.L., Jiang, H., Lu, Q., Dai, S., Hancock, W.S., Karger, B.L.: Mass spectrometric determination of disulfide linkages in recombinant therapeutic proteins using online LC-MS with electron-transfer dissociation. Anal. Chem. 81, 112-122 (2009)

33. Washburn, M.P., Wolters, D., Yates III, J.R.: Large-scale analysis of the yeast proteome by multidimensional protein identification technology. 19Nat. Biotechnol. 19, 242-247 (2001) 